\title{
VLT/FLAMES observations of Collinder 261
}

\author{
P. Spanò ${ }^{1,2}$ R. Pallavicini ${ }^{2}$ and S. Randich ${ }^{3}$ \\ ${ }^{1}$ DISF\&A, Univ. di Palermo, Piazza del Parlamento 1, I-90134 Palermo, Italy \\ email: spano@astropa.unipa.it \\ ${ }^{2}$ INAF - Oss. Astronomico di Palermo, Piazza del Parlamento 1, I-90134 Palermo, Italy \\ ${ }^{3}$ INAF - Oss. Astrofisico di Arcetri, Largo Fermi 5, I-50125 Firenze, Italy
}

\begin{abstract}
We report on results of VLT/FLAMES observations of the very old cluster Cr 261 . We compare the results with those of other clusters older than the Hyades.
\end{abstract}

Keywords. Stars: abundances, open clusters and associations: general

\section{Introduction}

The study of open clusters has received a great boost with the advent of the multiobject spectrograph FLAMES at the VLT, due both to the larger telescope area and to the multiplexing capability. Open clusters are among the preferred targets for observations with FLAMES, since they are key tracers of stellar and Galactic evoution. In particular, they offer a unique opportunity for investigating Li depletion and nonstandard mixing mechanisms in stars during their main-sequence evolution (see reviews by Deliyannis 2000, Jeffries 2000, Pallavicini et al. 2004).

\section{The observations}

We have observed with FLAMES the open clusters NGC2506 (age 2 Gyr, Marconi et al. 1997), Melotte 66 ( $\sim 4$ Gyr, Kassis et al. 1997) and Cr 261 ( 7 Gyr, Gozzoli et al. 1996; Friel et al. 2003; Prisinzano et al. 2005). The spectroscopic data, obtained with the GIRAFFE spectrograph, were reduced with the standard pipeline and are being analysed to derive radial velocities, Li abundances, and metallicities for turn-off (TO) and lower MS stars. Preliminary results on Li abundances in Cr 261 are presented here. Results for the other clusters will be reported elsewhere (Spanò et al. 2005).

\section{Results for the old cluster Cr 261}

Cr 261 is one of the oldest open clusters in the Galaxy, with an estimated age ranging from $\sim 7$ to $\sim 9$ Gyr (Janes et al. 1994; Phelps et al. 1994; Gozzoli et al. 1996; Prisinzano et al. 2005). The metallicity, derived from both low-resolution and high-resolution spectra, is slightly subsolar (Friel et al. 2002; Friel et al. 2003) and the reddening is highly uncertain, with quoted $\mathrm{E}(\mathrm{B}-\mathrm{V}$ ) values ranging from 0.22 to 0.34 (Janes et al. 1994; Mazur et al. 1995; Gozzoli et al. 1996; Prisinzano et al. 2005; see however Bragaglia et al. 2005 for different results). Our radial velocity analysis has identified 138 likely cluster members, out of 220 observed stars, with a maximum of 9 possible spurious objects. Li abundances (Fig. 1) show a spread nearly as large as the one in M67 (Jones et al. 1999) except for the fact that the upper limits in Cr 261 are significantly higher than in the latter cluster. The derived maximum $\mathrm{Li}$ abundances for solar-type stars are comparable to those measured in the very old ( 6-8 Gyr) cluster NGC 188 (Randich et al. 2003) and in the 


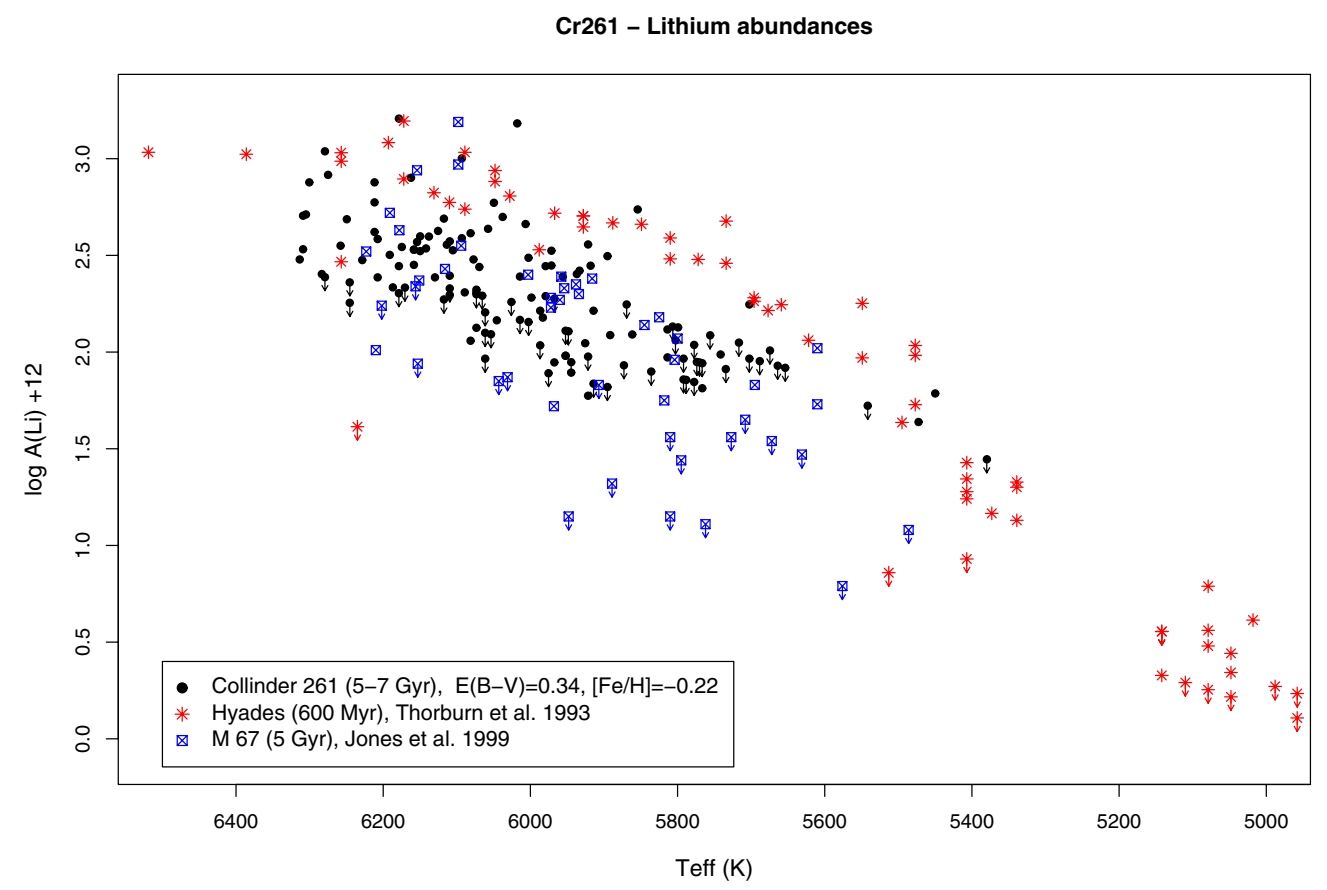

Figure 1. Li abundances vs. $\mathrm{T}_{\text {eff }}$ for MS stars in Cr 261.

upper envelope of the Li distribution of M 67, but are affected by a large systematic uncertainty due to the poorly determined reddening of the cluster. Comparison of the $\mathrm{Li}$ vs. Teff distribution of Cr 261 with those of the Hyades and M 67 suggests a high value of the reddening $(\sim 0.34)$ close to the highest value found in previous studies. A high-value of the reddening is also required to bring photometric and spectroscopic temperatures into agreement (cf. Friel et al. 2003).

\section{References}

Bragaglia, A. et al. 2005, A\&A, accepted

Deliyannis, C.P. 2000, Proc. ASP, IAU Symp. 198, 61

Friel, E.D. et al. 2002, $A J, 124,2693$

Friel, E.D. et al. 2003, AJ, 126, 2372

Gozzoli, E. et al. 1996, MNRAS, 283, 66

Janes, K.A. et al. 1994, AJ, 108, 1773

Jeffries, R.D. 2000, Proc. ASP, IAU Symp. 198, 245

Jones et al. 1999, $A J, 117,330$

Kassis, M. et al. 1997, AJ, 113, 1723

Marconi, G. et al. 1997, MNRAS, 291, 763

Mazur, B. et al. 1995, MNRAS, 273, 59

Pallavicini, R. et al. 2004, ESA, Special Publication, in press

Phelps, R.L. et al. 1994, AJ, 107, 1079

Prisinzano, L. et al. 2005, in preparation

Randich, S. et al. 2003, A\& A, 339, 133

Spanò, P. et al. 2005 in preparation

Thorburn et al. 1993 ApJ, 415, 150 